\title{
OBSERVATIONS OF RISING TUNE DURING THE INJECTION INSTABILITY OF THE IPNS RCS PROTON BUNCH *
}

\author{
J. C. Dooling ${ }^{\dagger}$, F. R. Brumwell, L. I. Donley, K. C. Harkay, R. L. Kustom, M. K. Lien, \\ G. E. McMichael, M. E. Middendorf, A. Nassiri, and S. Wang, ANL, Argonne, IL 60439, U.S.A.
}

\section{Abstract}

In the Rapid Cycling Synchrotron (RCS), a single proton bunch $(\mathrm{h}=1)$ is accelerated from $50 \mathrm{MeV}$ to 450 $\mathrm{MeV}$ in approximately $14.2 \mathrm{~ms}$. The bunch experiences an instability shortly after injection $(<1 \mathrm{~ms})$. During the first millisecond, the beam is bunched but little acceleration takes place; therefore this period is similar to a storage ring mode of operation. Natural vertical oscillations (assumed to be tune lines) show the vertical tune to be rising toward the bare tune value, suggesting neutralization of space charge and a reduction of its detuning effects. Neutralization time near injection ranges from $250 \mu \mathrm{s}-500 \mu \mathrm{s}$, depending on the background gas pressure. Oscillations move from the LSB to the USB before disappearing. Tune measurements made with a recently installed ferritemagnet pinger system show the horizontal chromaticity to be positive early but approaching zero later in the cycle; on the other hand, the vertical chromaticity is negative throughout the cycle. During pinger studies near injection, two vertical lines are observed. Neutralization of the beam space charge implies the generation of plasma in the beam volume early in the cycle which may then dissipate as the time-varying electric fields of the beam become stronger.

\section{INJECTION-COASTING BEAM}

In earlier discussions, it was argued that a significant population of electrons can be present in the RCS, especially during injection[1]; however, it has proven difficult to observe them in a repeatable manner. During injection (and stripping) of the $50 \mathrm{MeV} \mathrm{H}^{-}$ion beam and for several hundred microseconds thereafter, protons in the RCS are only weakly accelerated as they are bunched. At this time, the RCS takes on some of the qualities of a storage ring and exhibits what appears to be an instability. An example of a vertical difference (top-bottom) BPM pie-electrode spectrum, centered approximately $80 \mu \mathrm{s}$ after the end of injection is presented in Figure 1; the time span of the sample is $40 \mu \mathrm{s}(\Delta \mathrm{f}=25 \mathrm{kHz})$. The large lower side-bands (LSBs) present in this spectrum are similar to those that have been observed at the PSR[2]. The SBs we observe are self-excited by the beam and not the result of any external force applied to the beam to induce oscillations (e.g., from pinger magnets for tune measurements). Never the less, as with pinger tune measurements, it is assumed that the observed SBs are indicative of the tune. Successive FFTs of pie data reveal

\footnotetext{
*Supported by DOE under contract number DE-AC02-06CH11357. †jcdooling@anl.gov
}

time behavior of important beam spectral components in the ring as shown in Figure 2. In this case, pie data, sampled at $1.25 \times 10^{9} \mathrm{Samples} / \mathrm{s}(1.25 \mathrm{GS} / \mathrm{s}, 8$-bit/S), is broken into windows of 8 - $\mu$ s duration $(12.5 \mathrm{kS})$. An FFT of each data window is performed and the amplitudes of both the fundamental RCS rf ring frequency $(\sim 2.2 \mathrm{MHz})$ as well as the fixed linac frequency $(200.07 \mathrm{MHz})$ components are shown near injection. The pie electrode is evidently sensitive to the microbunch frequency thus providing a convenient time marker for the data. The injected macro-bunch pulse-width is typically $70-80 \mu \mathrm{s}$ and delivers approximately $0.5 \mu \mathrm{C}$ of $\mathrm{H}^{-}$ions to the RCS ring $(h=1)$. Note that injection begins $220 \mu$ s before the time of minimum magnetic guide field, $t\left(B_{\min }\right)$, which is defined as $t=0$ for the acceleration cycle. In the post-

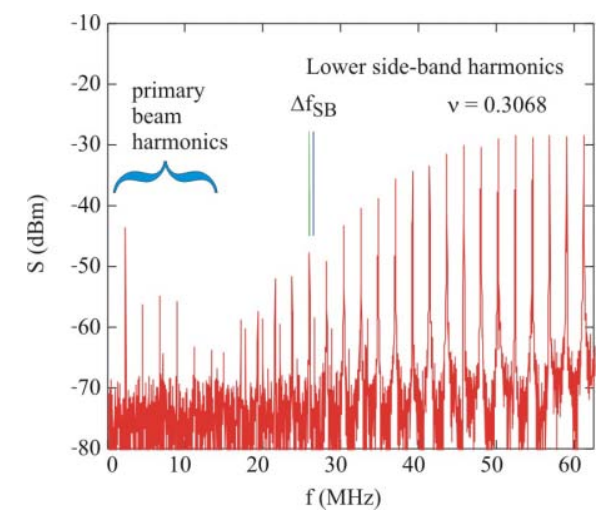

Figure 1: RCS vertical pie difference spectrum.

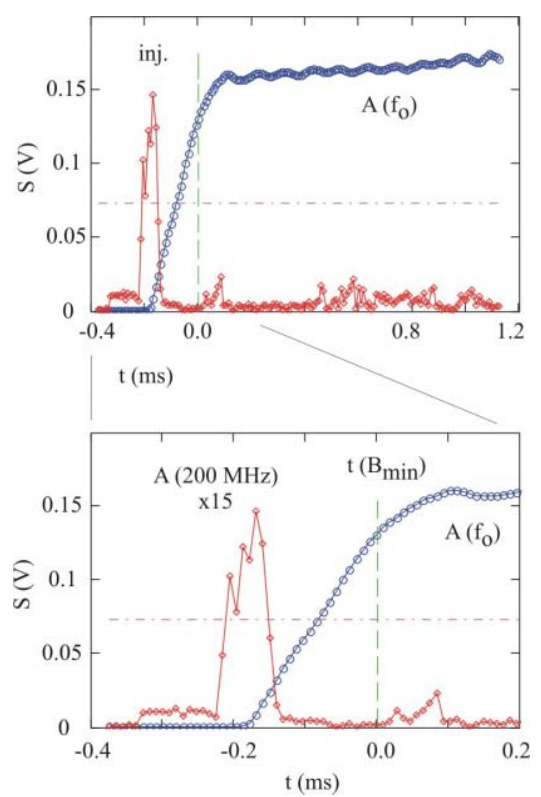

Figure 2: Injected and circulating spectral amplitudes. 
injection interval the frequency shift is less than 0.5 percent out to $\mathrm{t}=0.6 \mathrm{~ms}$, and by less than 2 percent for the first $1.2 \mathrm{~ms}$.

\section{TUNE MEASUREMENTS}

Using an optimized FFT approach to determine the frequency in a given time interval (the RCS fundamental frequency varies from $2.21 \mathrm{MHz}$ to $5.14 \mathrm{MHz}$ in 14.2 $\mathrm{ms}$ ). fractional tune values are obtained[3]. A ferrite pinger magnet pair was installed in the RCS ring to allow simultaneous $\mathrm{x}-$ and $\mathrm{y}$-tune and chromaticity measurements to be conducted at any time of interest during the acceleration cycle. Typically, a bunch of relatively low charge (80-120 $\mathrm{nC})$ is accelerated the ring for pinger tune measurements to reduce beam loss; however, full charge injection pinger studies have also been carried out to examine the effects of coherent spacecharge tune shift. A comparison of low-intensity (LI, 80 $\mathrm{nC})$ and full-intensity (FI, $0.5 \mu \mathrm{C})$ central-orbit vertical tune measurements, $1-\mathrm{ms}$ after injection show $v_{\mathrm{y}}=0.30$ for LI and $v_{\mathrm{y}}=0.28$ and for FI. MAD simulation predicts the horizontal and vertical bare tunes to be $\mathrm{Q}_{\mathrm{x} 0}=2.20$ and $\mathrm{Q}_{\mathrm{y} 0}=2.32$, and these values have been confirmed with pinger tune measurements.

The temporal behavior of self-excited, fractional vertical tune shortly after injection is plotted in Figure 3. The data labeled $0.6 \mu \mathrm{C}$ comes from six separate acceleration cycles in February 2004 when the RCS experienced its highest average operating current, near $16.5 \mu \mathrm{A}$. Also shown in Figure 3 is a single LI case (also self-excited). After a period of rise, the fractional frequencies (vertical tune) in the high intensity cases drop back to the measured space-charge shifted values from pinger measurements. This implies that the electrons initially trapped in the beam have been expelled probably through the bunching process.

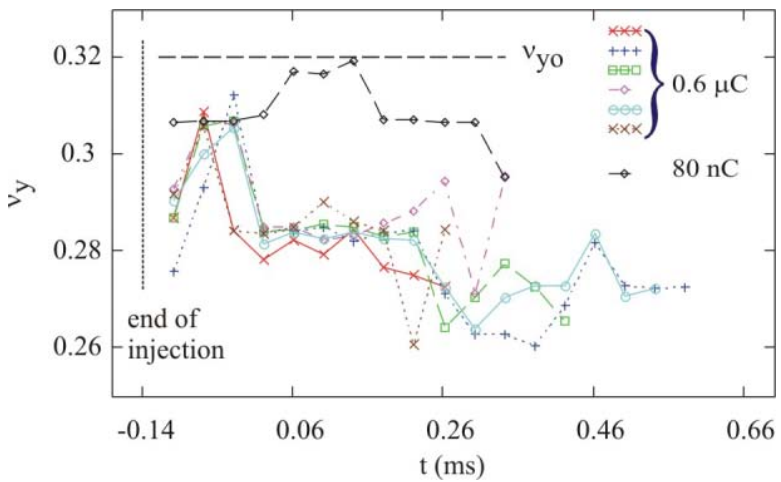

Figure 3: Self-excited, fractional vertical tunes shortly after injection

\section{IONIZATION AND NEUTRALIZATION}

\section{Beam Ionization}

Ionization cross sections and neutralization times in the RCS have been previously discussed[1]. Near injection, the DC neutralization time in the RCS varies from $250 \mu \mathrm{s}$ to $500 \mu$ s assuming $\mathrm{N}_{2}$ gas at pressures of $2 \mu$ Torr and 1 $\mu$ Torr, respectively. Background gas pressure in the RCS vacuum chamber typically averages $1.5 \mu$ Torr; however, actual measurements can vary by a factor of 4 depending on location in the ring. The production rate of species $\alpha$ is expressed as,

$$
\mathrm{R}_{\alpha}=\mathrm{n}_{\mathrm{i}} \mathrm{n}_{\mathrm{j}}\left\langle\sigma_{\alpha}\left(\mathrm{v}_{\mathrm{ij}}\right) \mathrm{v}_{\mathrm{ij}}\right\rangle=\mathrm{n}_{\mathrm{i}} \mathrm{n}_{\mathrm{j}} \sigma_{\alpha \mathrm{o}} \beta \mathrm{c}
$$

where $\mathrm{n}$ is density, $\sigma_{\alpha}\left(\mathrm{v}_{\mathrm{ij}}\right)$ is the velocity-dependent cross section and indices $\mathrm{i}$ and $\mathrm{j}$ refer to incident (beam) and target (background gas) components. For a monoenergetic beam incident on a stationary target, the implied integration in Equation 1 occurs over the delta-function in velocity space, and the cross-section is just the value of the function at the beam velocity $\beta(=\mathrm{v} / \mathrm{c})$. The electron production rate for the full acceleration cycle as well as the electron density generated per turn is presented in Figure 4. These electrons, trapped in the potential of the beam then cause neutralization of the beam space charge reducing the magnitude of the coherent tune shift[4,5]. The density of electrons produced per turn is just the product of the production rate and the beam pulse width,

$$
\mathrm{n}_{\mathrm{e}}(\mathrm{t})=\mathrm{R}_{\mathrm{e}}(\mathrm{t}) \tau(\mathrm{t})
$$

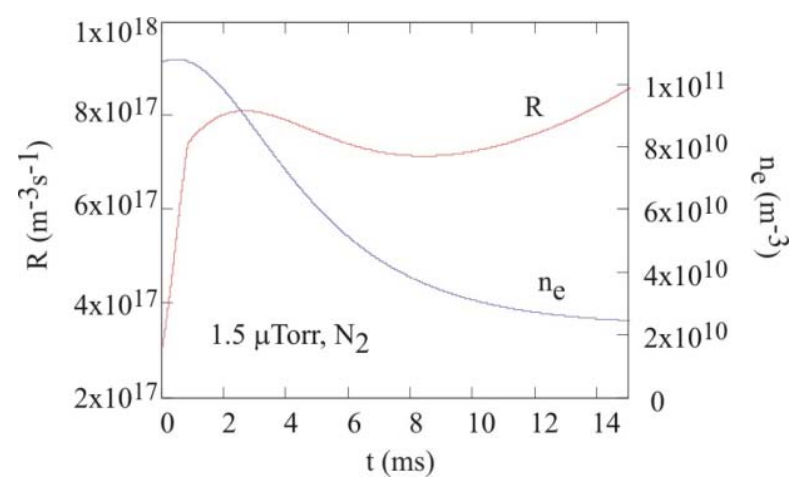

Figure 4: RCS electron production rate and density per turn

\section{Electron ionization}

The proton beam forms a potential which traps electrons when the beam is present. Electric field and potential profiles are given in Figure 5 for several times in the acceleration cycle, assuming a round proton beam of radius $1.5 \mathrm{~cm}$ in beam pipe of $3.8 \mathrm{~cm}$ inner radius. The charge is each case is $0.5 \mu \mathrm{C}\left(3.2 \times 10^{12}\right.$ protons $)$. The initial ion-electron population is created by the incident protons striking background gas atoms or molecules. The positive beam space-charge drives out the positive ions, leaving electrons which grow in number; however, this neutralization process alone does not appear to be fast enough to explain the early rise in the tune. Neutralization might be enhanced by the ionizing action 

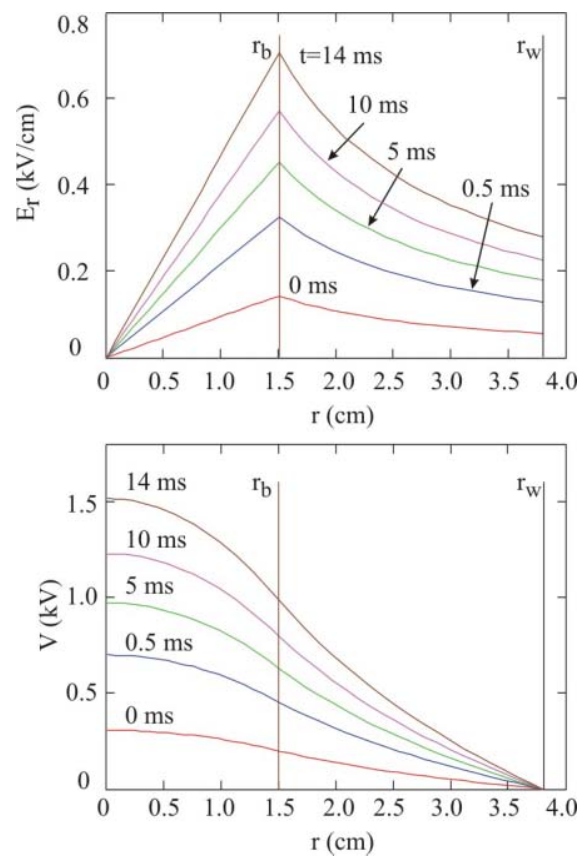

Figure 5: RCS radial electric field and potential profiles.

of the primary electrons. $50 \mathrm{MeV}$ protons have roughly the same ionization power as do $27 \mathrm{keV}$ electrons $\left(\beta_{\mathrm{p}}=\beta_{\mathrm{e}}\right.$ $=0.316$ ). After being liberated by ionization of the background gas, primary electrons will then oscillate in the space charge of the proton beam. In the round beam model described above, the beam-edge to beam center potential is $120 \mathrm{~V}$. Assuming the electrons are liberated with relatively low energy $(<50 \mathrm{eV})$, they will remain trapped in the potential well of the ions; additionally, the electrons now have energies near that yielding the maximum ionization cross section[6]. Initial electroninduced ionization rates, though lower than beam driven ionization, are proportional to the square of the background density. However, even at $10 \mu$ Torr, the electron contribution in one turn is less than direct proton ionization by a factor of roughly 27 . The background pressure would have to be raised to approximately 270 $\mu$ Torr before the contributions are approximately equal. Note that at $270 \mu$ Torr of $\mathrm{N}_{2}$, the primary electron density would reach $2.9 \times 10^{13}$ in a single turn, with a roughly equal density provided by electron ionization. The fractional neutralization level would then be approximately $\eta=2\left(2.9 \times 10^{13}\right) / 1.22 \times 10^{14}=0.47$. Such pressures may be obtained locally in transient events but do not occur over any significant fraction of the chamber; therefore, if neutralization is the cause for the rising tune in the RCS, another mechanism must occur to enhance the process.

\section{Density Enhancement}

From a transverse point of view, the electron ionization process should cause progressively lower temperature electrons to fall radially into the center of the beam. Initially, the electron velocity profile will peak at $r=0$ and fall to zero at the beam edge. Central electron density peaking is predicted for short positively-charged beam bunches in the presence of an electron cloud[7]. In the present case of a long bunch, the density of primary electrons from residual gas ionization will also peak near the beam center; the degree of peaking is dependent on the electron temperature. Though electrons may lose energy as they fall towards the center through collisions, they will still be accelerated by the longitudinal electric field of the proton beam. The enhanced central electron density, energized by the longitudinal field of the beam, may lead to further ionization of the background gas and a shortened neutralization time. This might suggest the center of the chamber becomes neutralized first; whereas at higher radii, the beam remains largely unneutralized.

\section{DISCUSSION}

Near the tune peak shown in Figure 4, approximately $100 \mu \mathrm{s}$ after the end of injection, the LSB exhibits two distinct lines as shown in Figure 6. Each peak is fit with a Lorentzian line shape to localize the frequency and tune. At this time the SB with greatest separation from the beam harmonic $(n=31)$ begins diminishing in amplitude. As bunching proceeds, trapped electrons are potentially being expelled from the beam volume.

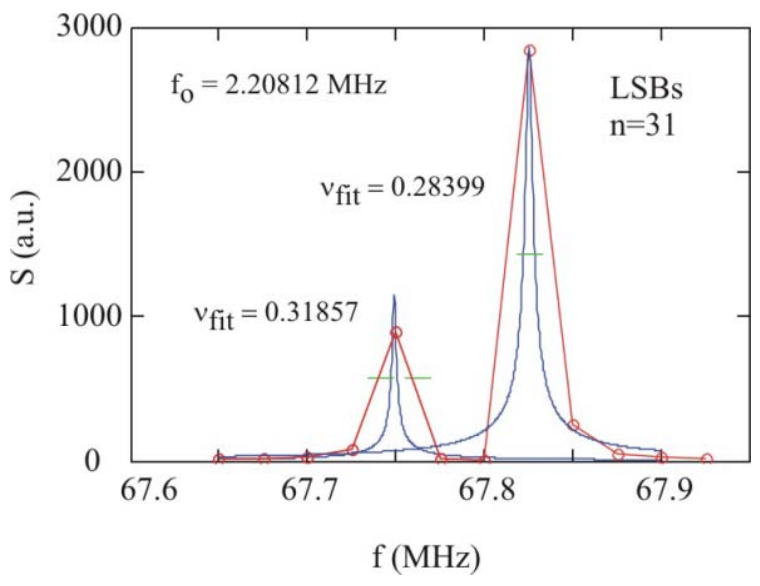

Figure 6: Two distinct LSBs near the tune peak

\section{REFERENCES}

[1] J. C. Dooling et al., Proc. PAC2003, Portland, IEEE, Piscataway, NJ, 2003, p. 592

[2] M. A. Plum, et al., Proc. PAC97, Vancouver, IEEE, Piscataway, NJ, 1997, p. 1611.

[3] J. C. Dooling, et al., BIW 2006, Fermilab, AIP 868, p. 281.

[4] Y. Baconnier, CERN Rpt. 85-19 (1985), p. 291.

[5] D. Neuffer et. al., NIM. A321, (1992) p. 1.

[6] M. Reiser, Theory and Design of Charged Particle Beams, Wiley, New York, 1994, p. 274.

[7] G. Rumolo, et al.,PRST-AB 6, 081002 (2003).

D04 Instabilities - Processes, Impedances, Countermeasures 